\title{
Curriculum Management and CEAB Outcome Reporting: Recent Activities at the University of British Columbia
}

\author{
S. Nesbit*, S. Wilton, A. Ivanov, T. Froese, R. Sianchuk \\ Faculty of Applied Science, University of British Columbia \\ *nesbit@mail.ubc.ca
}

\begin{abstract}
Since 2010, the departments of Civil Engineering, and Electrical and Computer Engineering have partnered in the development of a program assessment protocol aimed at determining how well graduating students achieve independently created Program Learning Goals (PLGS). More recently, the departments are working together to prepare for CEAB Accreditation visits in 2014. This has been a fruitful partnership in part because of the very different undergraduate engineering programs offered by the two departments.

This paper reports the curriculum management approach that has emerged from the collaboration between the two engineering departments, data that has been collected to test hypothesized assessment protocols, and results from a pilot data collection process developed for CEAB outcomes and continual improvement purposes. The paper highlights the management approach, which is based on the conceptualization of the engineering programs as socio-curricular systems, the development of PLG indices, and results from the pilot CEAB outcomes reporting that involves the collection of triangulated data, i.e. the collection of data from the faculty perspective, the student perspective, and the perspective of the Professional Engineering community.
\end{abstract}

Keywords: Curriculum Management, CEAB Reporting, Planned Curriculum.

In 2008-2009, the Departments of Electrical and Computer Engineering (ECE), and Civil Engineering (CIVL), at the University of British Columbia, independently developed Programme Learning Goals (PLGs) for their undergraduate curricula and, since 2010, the departments have partnered in the development of a program assessment protocol aimed at determining how well graduating students are achieving the PLGs. This work resulted in the development of a curriculum management approach, and a pilot of a curriculum assessment protocol based on the Analytical Hierarchy Process (AHP).
More recently, the departments are working together to report outcome-based assessment results to CEAB in 2014. The following research questions are posed: (i) does the curriculum offer constructively aligned learning opportunities for students to develop the CEAB attributes? (ii) what curricular resources support student development of the attributes? (iii) to what extent does the graduating class exhibit and demonstrate the $\mathrm{CEAB}$ attributes?

The presentation starts by describing the engineering education enterprise as the aim of an adaptive system made of multiple interacting components (see Figure 1). An overarching approach to curriculum management is proposed wherein curriculum monitoring, assessment, and reporting play pivotal roles in engaging the learning community and informing management decisions. It then proposes a monitoring system involving collection of triangulated data aimed at answering the research questions.

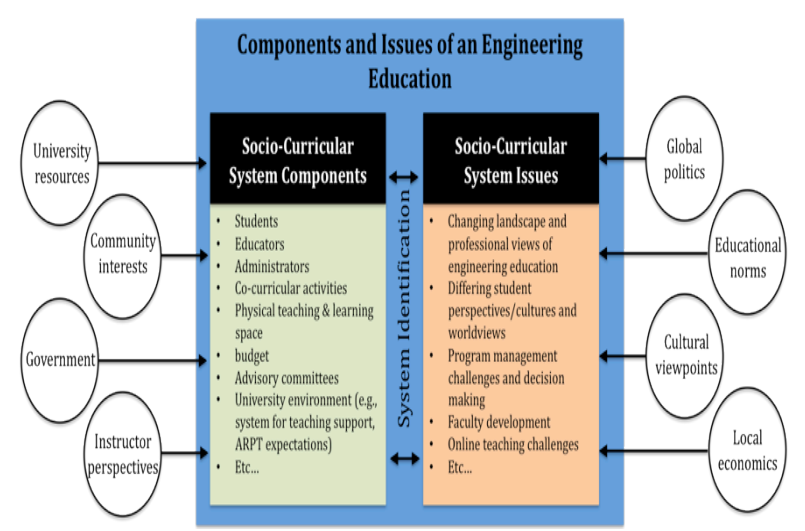

Fig. 1. The engineering education enterprise as the focus of a socio-curricular system.

Finally, initial results from pilots of data collection activities are presented. The presentation closes with a brief reflection on what has been learned to date about developing and applying curriculum assessment in practice. 